\title{
Multipurpose monitoring system for icebreakers: Development, implementation, and testing
}

\author{
Andrey Zhirnov, Maxim Anufriev, Nikolay Pozhar, Konstantin Stepanov, Anton Chernutsky, llya Makhrov, Evgeniy Nesterov, \\ Dmitriy Shelestov, Kirill Koshelev, Aleksey Fedorov, Valeriy Karasik, and Alexey Pnev
}

Bauman Moscow State Technical University, 105005 Moscow, Russia

\begin{abstract}
We demonstrate the results of a project on implementing a complete and practical multipurpose monitoring system for icebreakers. We describe the basic principles of operating the developed monitoring system. We present a 3D finite element analysis of the ship model to optimise the sensor arrangement. We also present the benchmarks and test results of the system during an expedition on the Russian scientific research vessel Akademik Tryoshnikov.
\end{abstract}

\section{Introduction}

The development of solutions for the structural health monitoring of complex objects such as buildings, bridges, and ships is a challenging problem [1]. It has been the subject of active study for the last several decades [2]-[5].

In particular, monitoring systems are very important for icebreakers and seagoing ships. Because the cost of technical service of such objects is significantly high, problems related to the design of monitoring systems for accident prevention have attracted a great deal of interest. Note that obtaining accurate data regarding ship structural health is key for route planning optimisation. Currently available solutions are not universal because they are used to control a limited number of parameters only [6-12]. Light Structures AS (Norway), SENSFIB project, monitors the ship hull in real time. It analyses strain continuously and generates emergency messages with advices to the stuff when safe limits are exceeded. BMT Scientific Marine Services Inc. (USA), Structural Monitoring System, designed mainly for monitoring of offshore structures (offshore drilling platforms, including ice-resistant) and also mooring systems. Weir-Jones Ltd (Canada), Ice Force IDEAL, designed for monitoring of the interaction between ice and ship,measuring its parameters and for the protection of offshore platforms from the ice loads. Some gravity type ice-resistant platforms in Caspian Sea are equipped with this system. MCA Consulting (USA), Hull Monitoring System, designed mainly for monitoring of offshore structures. HULLMOS systems was installed on a five oil tankers of the project Sakhalin-1. Sea Structure Technology Co. Ltd (Korea), Naviscan, and Strainstall UK Ltd (UK), StressAlert II, designed for monitoring of the interaction between ice and ship.
We also note that there are no multipurpose systems for monitoring of the hull structure and the shafting and steering module altogether.

A number of technologies under active development, such as Supervisory Control and Data Acquisition (SCADA) [3] and wireless sensor networks [4], have attracted interest as a basis for monitoring systems. The aim of the current project was to develop a system for multipurpose monitoring of an object with the following advantages:

1) It contains all the latest achievements of the analogues.

2) Gives a hall monitoring parameter set from four hull monitoring subsystems and two shafting and steering module monitoring subsystems.

3) Uses fiber optic sensors for increasing the number of control points and decreasing the connection cable length.

4) The ability of system scaling for large and small structures.

5) For Russian companies it is more rapid and comfortable communication with the developer then in case of foreign implementer.

\section{Multipurpose monitoring systems}

The monitoring system provides complex control of the state of the object by measuring a number of parameters. The system consists of subsystems. Fig. 1 presents a complete and detailed decomposition of the monitoring system into subsystems as well as a scheme of the sensor arrangement.

We can decompose our monitoring system into six subsystems. Three subsystems control local (fibre Bragg sensors) and global (GPS/GLONASS, gyroscopes, accelerometers) deformations. The fourth subsystem using acoustic emissions to monitor cracks in the object. 
The fifth subsystem controls the shafting and steering module. Finally, the last subsystem integrates with preinstalled monitoring systems in the object. This subsystem allows general information about the situation to be visualised on the operator desk during an expedition. It typically includes control of the ice around the ship, a meteorological summary, information about navigation, dynamical parameters (such as speed), fuel consumption, and timeframes for the operation of different systems on the ship.

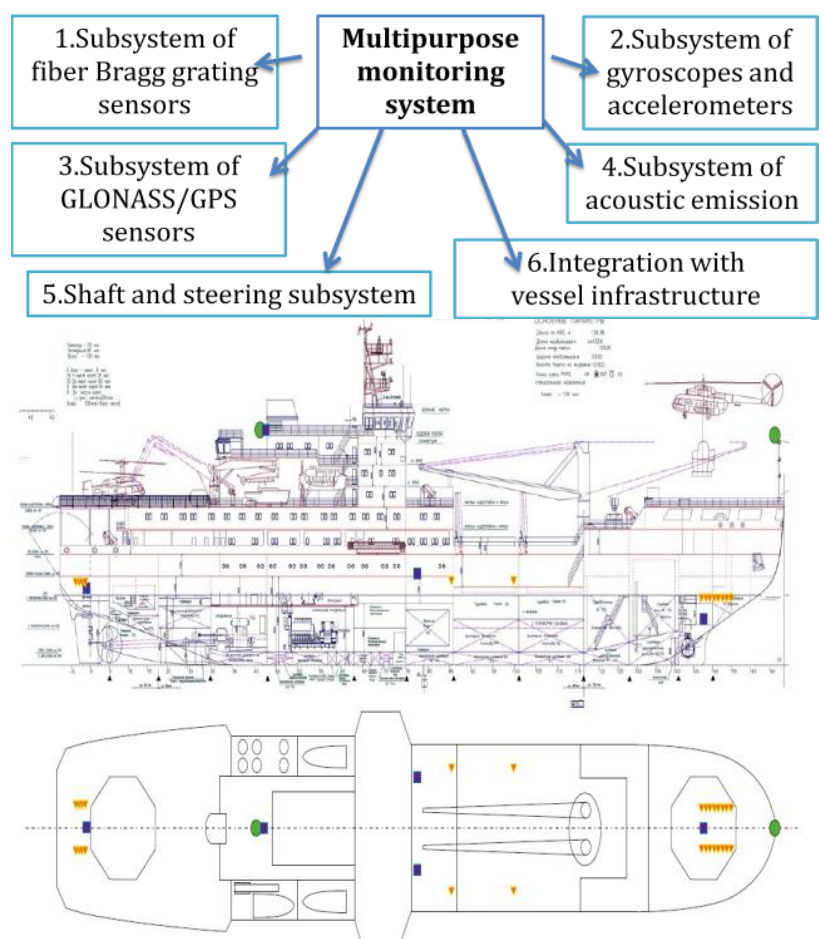

Figure 1. (Upper) Decomposition of the system. (Bottom) Structure and arrangement schemes of different types of sensors. Green circles: GLONASS/GPS sensors, violet squares: gyroscopes and accelerometers, yellow triangles: strain sensors.

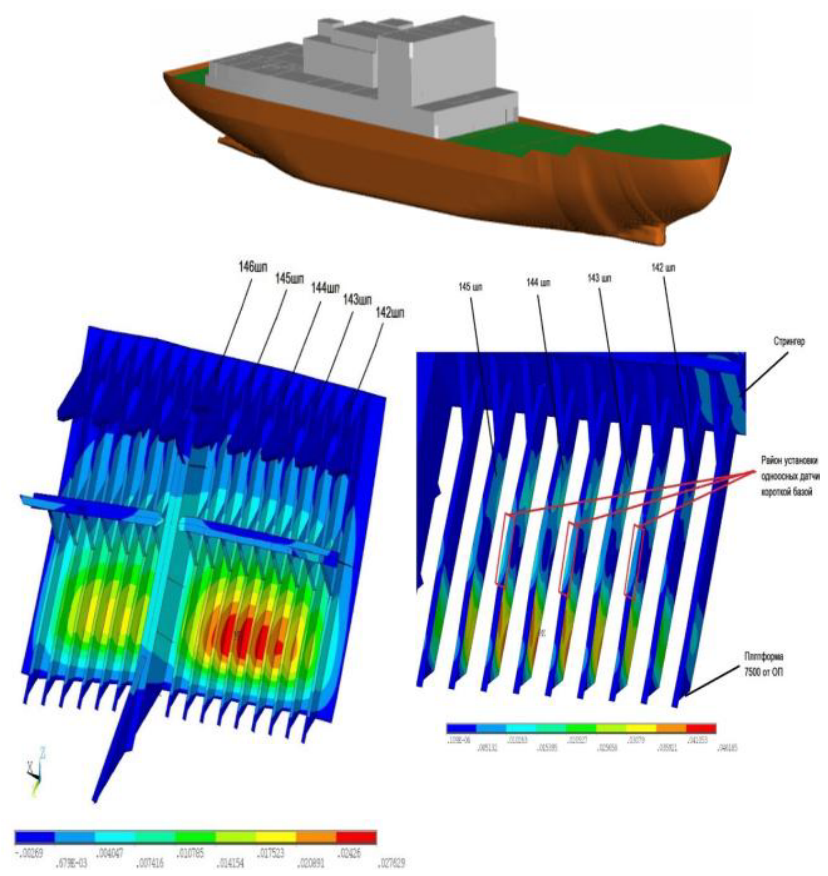

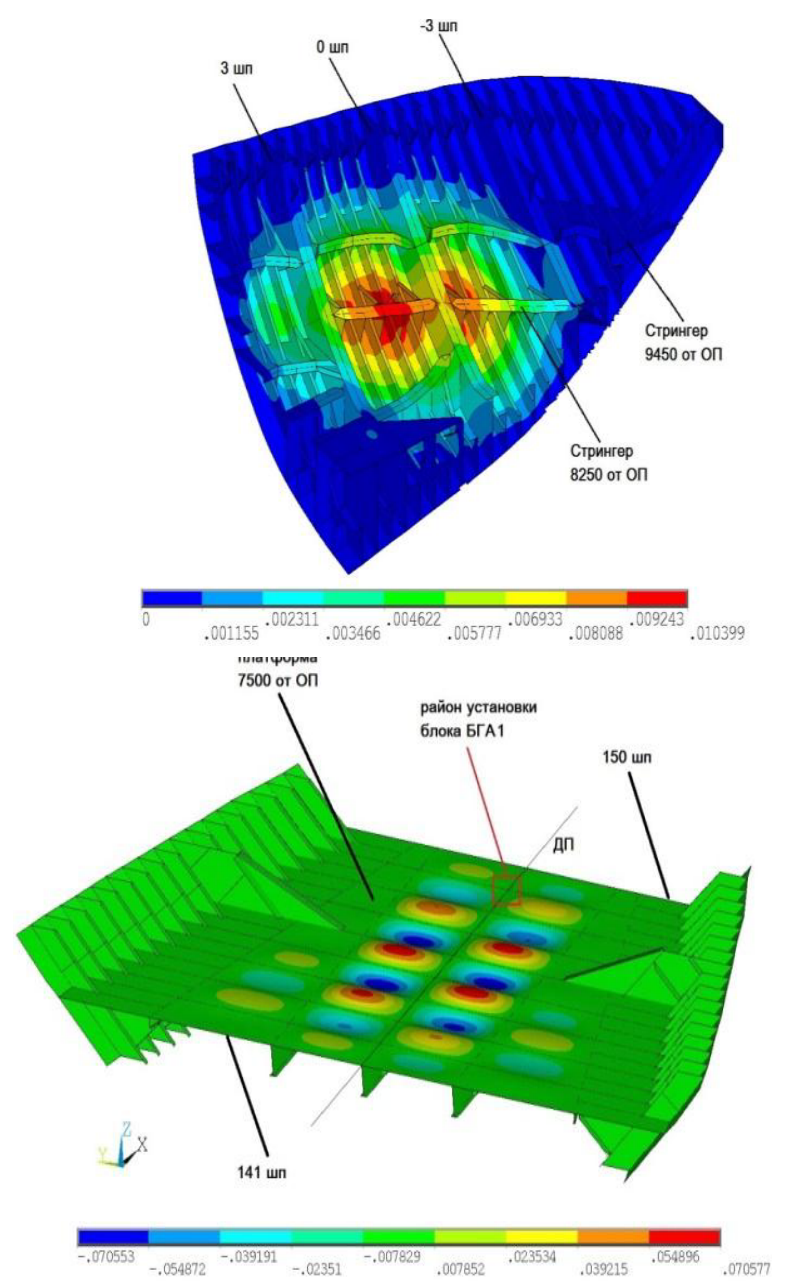

Figure 2. 3D numerical simulation results for different parts of the scientific research vessel Akademik Tryoshnikov.

Special attention should be paid to the monitoring of the hull construction. The monitoring workflow is based on the work of several subsystems. The gyroscope and accelerometer subsystem measures global deformations of the hull, such as displacements and rotations of the bow. The acoustic subsystem controls the construction integrity, i.e. it prevents crack formation by measuring acoustic pictures. The fibre-based sensor subsystem controls local deformations in different parts of the object. The GPS/GLONASS subsystem monitors longitudinal deformations.

\section{Numerical simulations}

The first steps of the project involved determining the optimal position for all types of sensors. In order to do this, we performed numerical simulations of the ship. The developed model of the object, which was chosen for an expedition in later stages of the project (see Section 5), allows all subsystems to be optimally located.

The model was designed in two basic stages. First, a geometric (CAD) three-dimensional model was created and analysed with the engineering software ANSYS. Second, a geometrical model of the object was analysed in ANSYS with the finite-element method. 
Table 1. Model characteristics before optimisation.

\begin{tabular}{|c|r|}
\hline Number of elements & 2645259 \\
Number of nodes & 2636014 \\
Degrees of freedom & 15816084 \\
\hline
\end{tabular}

Table 2. Model characteristics after optimisation.

\begin{tabular}{|c|r|}
\hline Number of elements & 797974 \\
Number of nodes & 740359 \\
Degrees of freedom & 4442154 \\
\hline
\end{tabular}

Special attention was paid to the problem of model optimisation. A large number of intersection lines for internal partitions of the vessel and the outer skin resulted in a large number of surfaces and boundaries with complex shapes. This complicated the creation of a finite element mesh with acceptable quality. Note that we excluded the vessel superstructure and its internal partitions, and some stiffening ribs were reduced to a common stiffness coefficient.

These assumptions allowed us to successfully address this problem without significant computational resources. Tables 1 and 2 present the achieved results.

To construct the finite-element model, three- and four-node shell elements having six degrees of freedom at each node (three translational and three rotational) were used. Each element was about $0.2 \mathrm{~m}$ in size. Fig. 2 presents the results of the numerical simulation.

\section{Implementation}

Each subsystem has its own processing block, which transmits data to the central server of the monitoring system. This server queries all measurements and allows any PC or notebook connected to the system network to access them.

Because the system was installed on the vessel, we had to follow some special requirements. First, all processing blocks were designed in accordance with the IP66 Rating Relationship to Humidity Tolerance.

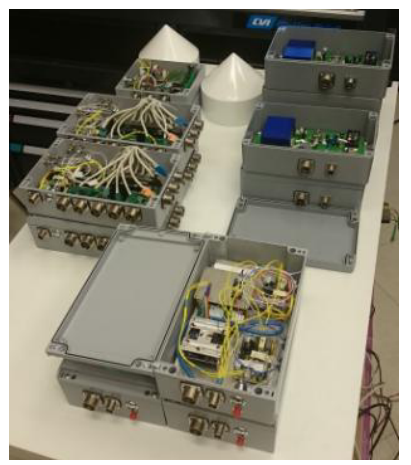

Figure 3. Part of the equipment in IP66 unit cases before being mounted on the vessel.

Note that the fibre-optic cables were halogen-free and flame-retardant, even though this is less safe for fibre splices. Finally, because an icebreaker strongly shakes during its expedition, all devices, sensors, and connection cables needed to be firmly fixed to the installation sites.

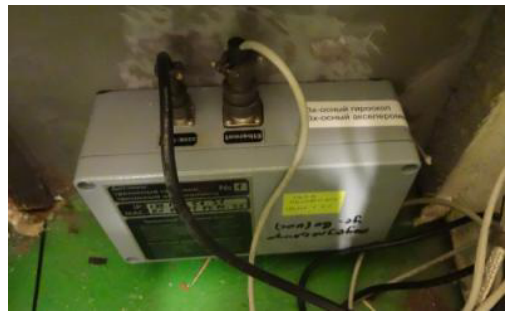

Figure 4. Mounted block of the gyroscope and accelerometer subsystem.

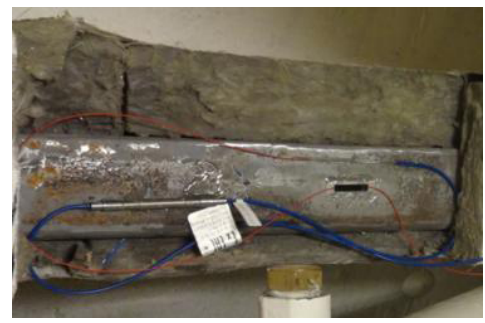

Figure 5. Mounted fibre Bragg grating strain sensor with the temperature compensator.

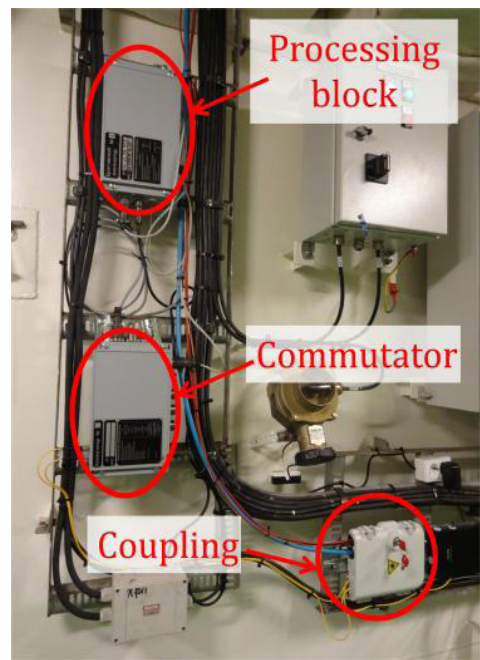

Figure 6. Mounted block of the subsystem of fibre Bragg grating strain sensors.

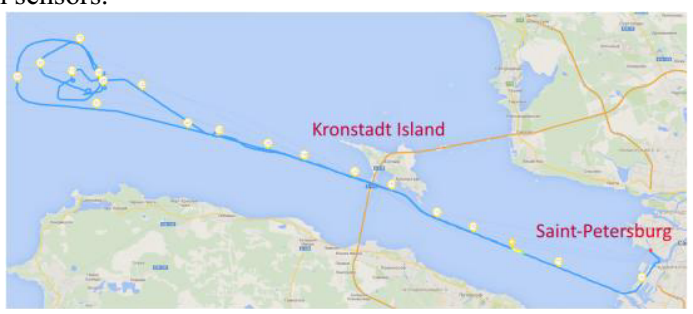

Figure 7. Expedition map.

\section{Expedition: Benchmarks and tests}

The above monitoring system was installed on the Russian scientific diesel-electric research vessel Akademik Tryoshnikov. All subparts of the monitoring system were installed (see Fig. 1) in the nose and tiller parts (strain gauges, accelerometers, gyroscopes), on the decks (antennas GLONASS/GPS), and tween-decks (strain gauges, accelerometers, gyroscopes, and sensors for acoustic-emission diagnostics). 
To collect benchmarks of multipurpose monitoring systems, the systems were tested over a two-day expedition (29 February-1 March 2016) on Akademik Tryoshnikov. Fig. 7 presents a map of the expedition.

The vessel was tested under different driving modes: forward and reverse, direct and circular vessel movement, various ice conditions and clean water, and different speeds.

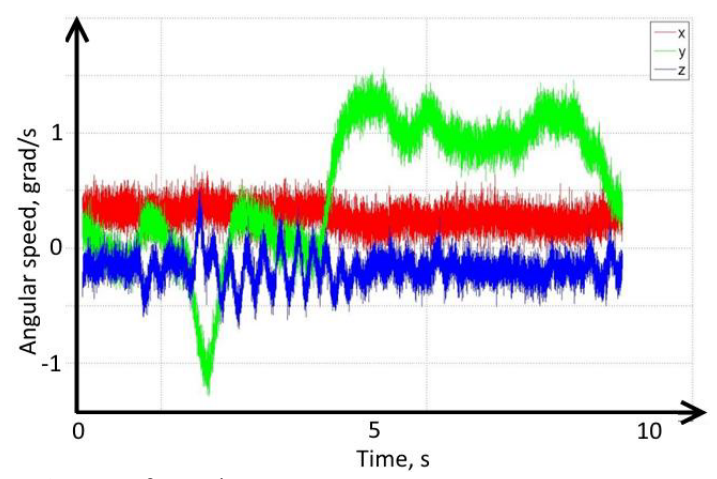

Figure 8. Data from the gyroscope sensor.

Fig. 8 shows an example signal from the gyroscope sensor from the tween-decks, which clearly shows the vessel incline during circulation. Fig. 9 shows the results of the GLONASS/GPS subsystem. A possible accuracy of $2 \mathrm{~cm}$ was confirmed. The moment of the ship impacting the pier was clearly recorded at 19:30. Fig. 10 presents the signals from the fibre Bragg sensor subsystem. The impact with the pier was also observed. Note that our system uses the method for pre-installed sensor deformation, which is described in [13].

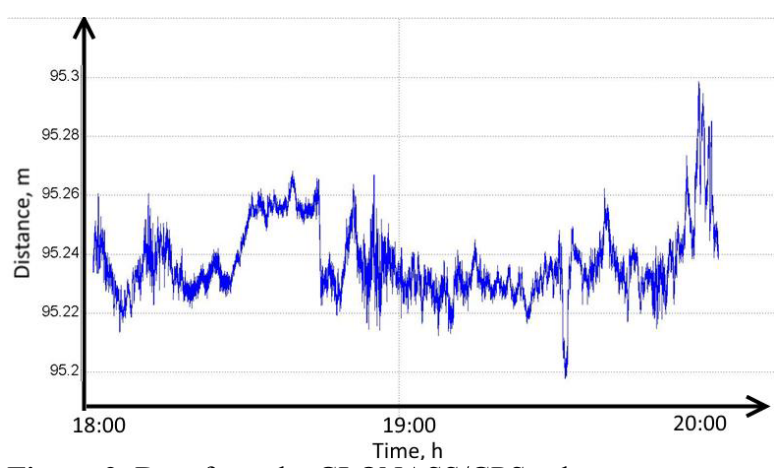

Figure 9. Data from the GLONASS/GPS subsystem.

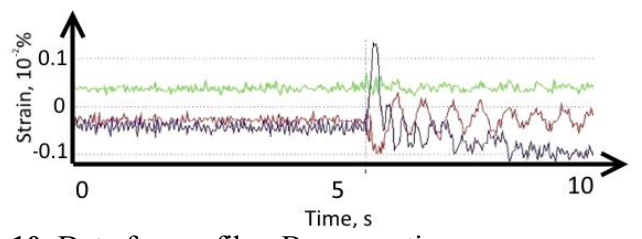

Figure 10. Data from a fibre Bragg grating sensor.

The data arriving at the processing server were recorded on data carriers. Based on the collected data, the behaviour of the object during the expedition can be traced. At this time, additional attention should be paid to the moment when the vessel struck the pier.

\section{Conclusions}

A current trend in ocean engineering is the installation of structural health monitoring systems on ships. These prevent accidents, which substantially reduces the cost of technical support.

This paper presents the results of a project on implementing a complete and practical multipurpose monitoring system for ships. Basic operating principles of the developed monitoring system are described along with the results of a 3D finite element analysis of the ship model. The benchmarks and test results of the system during a two-day expedition on Akademik Tryoshnikov are presented. The GLONASS/GPS subsystem for largescale deformation measurement and fibre Bragg subsystem using the compensation algorithm for local deformation measurement were successfully tested. Gyroscopes and accelerometers were useful to identify linear and angular accelerations of different ship parts. Shafting and steering monitoring subsystem measurements were used for vessel motion parameter calculations in different ice loads.

In June and July of 2016 is planned more significant tests of monitoring system during Arctic expedition. Russian fleet has many ice-class ships and platforms, so successful benchmarks may be promising for the massive usage of our system.

\section{References}

1. Bao, X., Chen, L. "Recent progress in distributed fiber optic sensors.” Sensors 12 (2012): 8601-8639.

2. Sielski, R.A. "Ship structural health monitoring research at the Office of Naval Research." JOM 64 (2012): 823-827.

3. Zaghloul, M.S. "Online ship control system using Supervisory Control and Data Acquisition (SCADA)." International Journal of Computer Science and Application 3 (2014): 6-10.

4. Xiong, X.-Y., et al. "Vibration monitoring system of ships using wireless sensor networks." IEEE International Conference on Mechatronics and Automation (ICMA), 902014.

5. Decò, A., Frangopol, D.M. "Real-time risk of ship structures integrating structural health monitoring data: Application to multi-objective optimal ship routing." Ocean Engineering 96 (2015): 312-329.

6. Structural Monitoring System http://www.scimar.com/

7. SMARTSTRESS http://www.bmtseatech.co.uk/

8. SENSFIB http://www.lightstructures.no/

9. Hull Monitoring System http://www.mcaco.com/

10. Hullmos http://www.rrouvari.fi/

11. Naviscan http://www.sst21c.com/

12. StressAlert II http://www.strainstall.com/

13. Fedorov, A.K., et al. "Structural monitoring system with fiber Bragg grating sensors: implementation and software solution." Journal of Physics: Conference Series 594 (2015): 012049. 